\title{
RESONANT COUPLING TYPE MICROSTRIP LINE INTERCONNECT USING A BONDING RIBBON AND DIELECTRIC PAD
}

\author{
Masashi Hotta*, Yongxi Qian, and Tatsuo Itoh \\ Electrical Engineering Department \\ University of California, Los Angeles \\ 405 Hilgard Avenue, Los Angeles, CA 90095
}

\begin{abstract}
Resonant coupling type microstrip line interconnects using a bonding ribbon and dielectric pad have been designed and fabricated. The basic concept of this interconnect is the $L C$ serial resonance of the pad capacitor and ribbon inductor. Both numerical simulation and experiment reveal low return loss and high efficiency connection at the predicted resonant frequency region, which can be readily shifted to higher frequencies by tuning the structural parameters. Furthermore, improvement in bandwidth of the interconnect is demonstrated by using a pad with higher dielectric constant.
\end{abstract}

\section{INTRODUCTON}

Ribbon bonding has been widely used for interconnect or packaging of MMICs and T/R modules at microwave and millimeter-wave frequencies. To tolerate thermal expansion and discrepancies in chip or component size, a small gap is usually left between two substrates to be connected. Because a large part of present MMICs or modules are based on microstrip structures, efficient interconnect between microstrip lines through a gap has great

*On leave from Department of Electrical and Electronic Engineering, Ehime University, Matsuyama, Ehime 790-8577, Japan importance for fabrication of high performance systems [1]-[3]. As is well known, the coupling efficiency usually deteriorates at higher frequencies, due to the parasitic inductance of the bonding ribbon [3], and special bonding configurations such as the use of tuning stub with line width compensation have been reported [1].

In this paper, we propose a high-efficiency DC-free microstrip line interconnect which does not require the modification of the shape of the microstrip lines to be connected. The new structure consists of a single ribbon and a
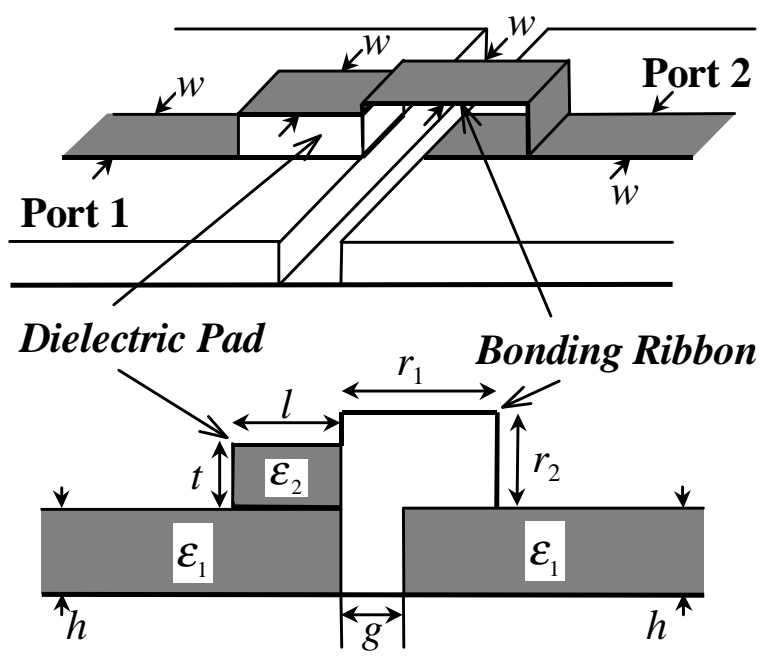

Fig. 1 Microstrip line interconnect through a gap using a bonding ribbon and dielectric pad. 
companion rectangular dielectric pad. The ribbon and the dielectric pad constitute a series $L C$ resonator so that high efficiency connection is achieved at the resonant frequency. Furthermore, a slight modification allows DC connection in addition to efficient coupling at the resonant frequency. We will present both numerical simulation and experimental verification of the coupling characteristics of this new interconnect structure. Our study also shows that improvement in frequency bandwidth can be realized by using a pad with higher dielectric constant.

\section{Microstrip Line INTERCONNECT}

Fig. 1 shows the proposed structure where two microstrip lines are connected through a gap with a metallic ribbon and a rectangular dielectric pad. The dielectric substrate for the $50 \Omega$ microstrip line used in this study is RT/Duroid with $\varepsilon_{1}=2.33$ and $h=0.8 \mathrm{~mm}$. The ribbon used is a rectangular copper sheet with $w=2.3 \mathrm{~mm}, r_{1}=4.0 \mathrm{~mm}$ and $r_{2}=1.4 \mathrm{~mm}$.

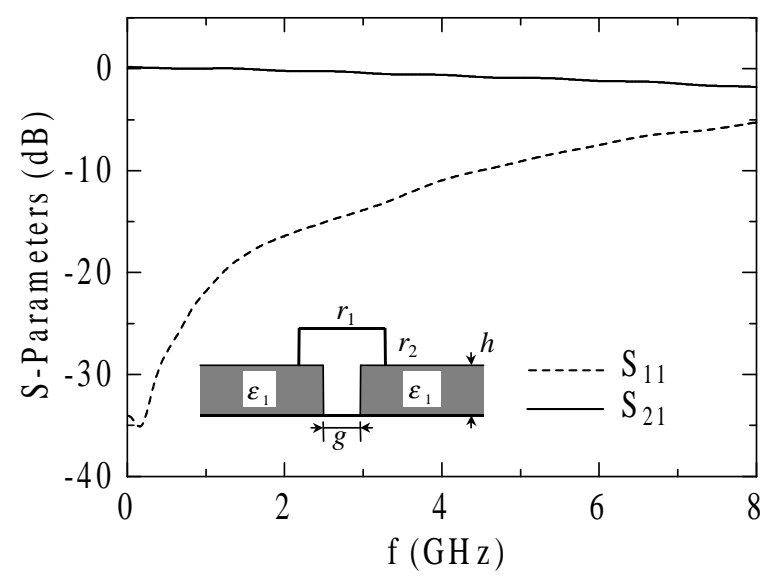

Fig. 2 Coupling characteristics of two microstrip lines connected by conventional ribbon bonding. ( $g=1.6 \mathrm{~mm}, h=0.8 \mathrm{~mm}, \varepsilon_{1}=2.33$, $r_{1}=4 \mathrm{~mm}, \quad r_{2}=1.4 \mathrm{~mm}$ and $w=2.3 \mathrm{~mm})$.
For the dielectric pads, two different kinds of materials with metallized top surfaces and identical widths to that of the ribbon have been investigated.

Fig. 2 shows the S-parameters simulated by FDTD for conventional ribbon bonding between microstrip lines through a gap of $g=1.6 \mathrm{~mm}$, which corresponds to the structure in Fig.1 without the dielectric pad. As expected, both insertion loss and return loss increase rapidly at higher frequencies. The deterioration of the ribbon bonding is mainly due to the parasitic inductance of the ribbon, which is estimated to be $3 \mathrm{nH}$ in the present case [4]. Hence, the introduction of a dielectric pad as shown in Fig. 1 will result in an $L C$ serial resonator, where an efficient coupling is expected around the resonant frequency.

\section{Simulation AND Measurement RESUlTS}

Fig. 3 shows both FDTD simulation and measurement results of the coupling characteristics of an interconnect using a dielectric pad with $\varepsilon_{2}=2.33, t=0.8 \mathrm{~mm}$ and $l=12 \mathrm{~mm}$. Due to the $L C$ resonance, the insertion loss is better than $0.8 \mathrm{~dB}$ from 3.0 to $3.8 \mathrm{GHz}$, and the return loss is below $-10 \mathrm{~dB}$ within this frequency range. Good agreement between measurement and prediction has been obtained, with a discrepancy of less than $3 \%$ for the resonant frequency.

Fig. 4 shows the FDTD simulated resonant frequencies for various lengths $l$ of the dielectric pad, with the other parameters identical to those in Fig. 3. Also shown in Fig. 4 is the estimated resonant frequency of the serial $L C$ resonator, where $L$ and $C$ are the ribbon inductance and pad capacitance, respectively [4]. The good correlation indicates that high-efficiency coupling should be possible at higher frequencies by reducing 


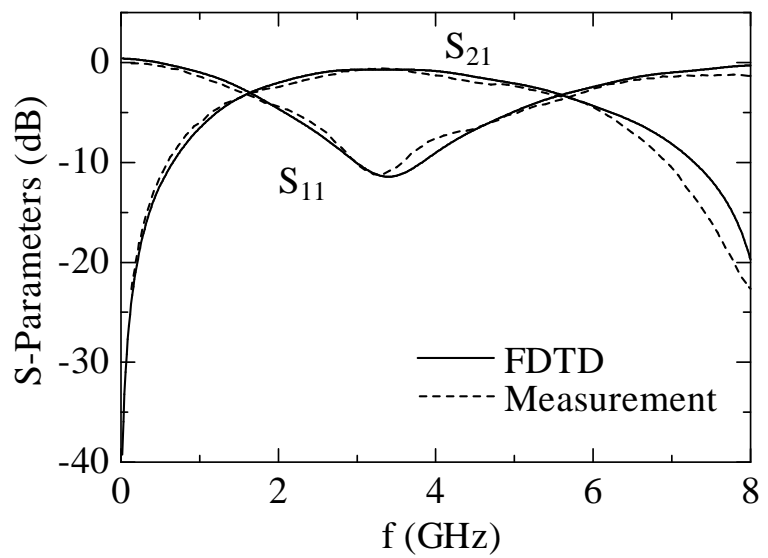

Fig. 3 FDTD simulation and measurement results of S-parameters for the microstrip line interconnect using bonding ribbon and dielectric pad with $\varepsilon_{2}=2.33, t=0.8 \mathrm{~mm}$ and $l=12 \mathrm{~mm}$. Other parameters are the same as in Fig. 2.

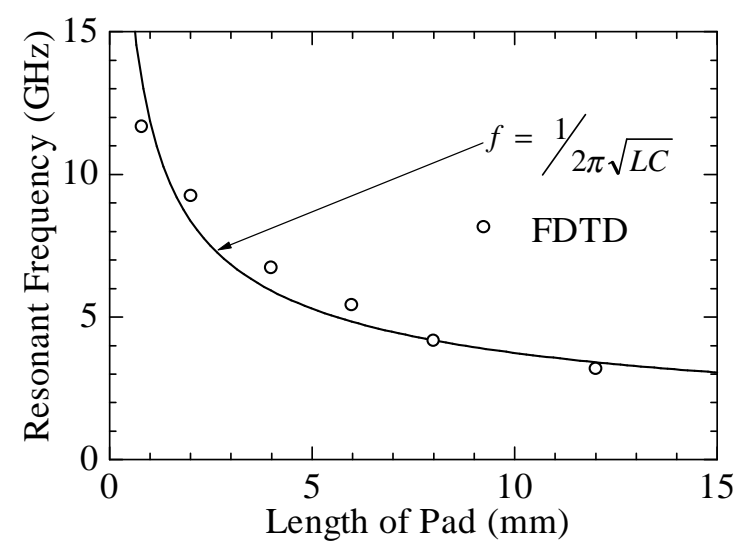

Fig. 4 Resonant frequencies of the interconnect with respect to different lengths of dielectric pad. Other parameters are the same as in Fig.3.

the size of the dielectric pad. The increasing discrepancy at higher frequencies also emphasizes the necessity of full-wave simulation at these frequencies.

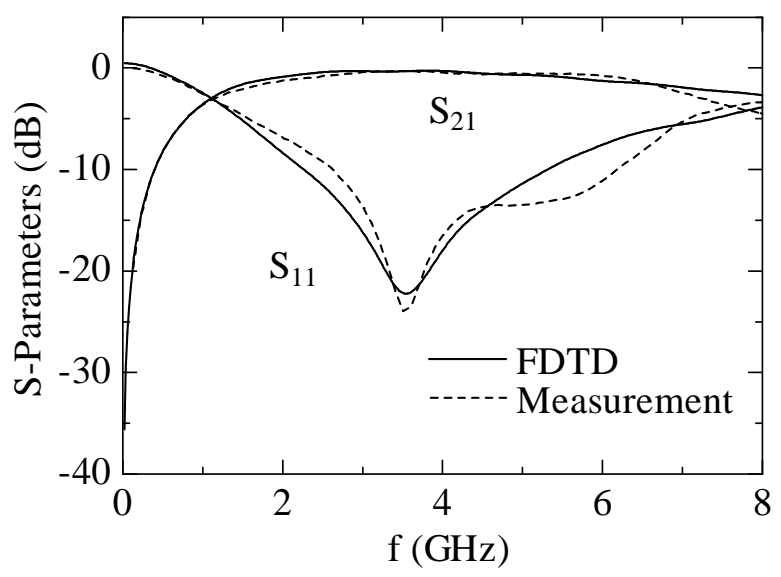

Fig. 5 Simulated and measured S-parameters of the microstrip line interconnect using bonding ribbon and dielectric pad with $\varepsilon_{2}=10.2$, $t=0.6 \mathrm{~mm}$ and $l=4 \mathrm{~mm}$. Other parameters are the same as in Fig. 2.

Fig. 5 shows the S-parameters for the interconnect using a dielectric pad with $\varepsilon_{2}=10.2, t=0.6 \mathrm{~mm}$ and $l=4 \mathrm{~mm}$. In this case, $S_{21}$ is better than $0.5 \mathrm{~dB}$ from 2.9 to $4.3 \mathrm{GHz}$, with the return loss below $-15 \mathrm{~dB}$. Again good agreement $(<2 \%$ discrepancy in resonant frequency) between theory and experiment is obtained. The results in Fig. 5 indicate that improvement in coupling efficiency and bandwidth is possible by using a thinner pad with higher dielectric constant.

\section{MOdifiEd STRUCTURE INCLUdING DC INTERCONNECTION}

In the previous section, resonant coupling type DC-free microstrip line interconnect has been presented. For some practical applications, however, an interconnect with DC pass is also required. The microstrip line on which the dielectric pad is loaded and the top conductor of a pad are connected to each other. This can be easily achieved by a slight modification of the proposed structure as shown 
in Fig. 6. S-parameters of this structure simulated by FDTD are shown in Fig. 7. The parameters are the same as in Fig. 3. Efficient coupling and low return loss interconnect $\left(S_{21}>\right.$ $-0.7 \mathrm{~dB}$ and $S_{11}<-15 \mathrm{~dB}$ ) are obtained from 6.0 to $7.0 \mathrm{GHz}$. From this result, it is expected that a high efficiency RF interconnection due to the $L C$ resonance together with DC pass can be realized.

\section{v. Conclusions}

In this paper, we proposed a new resonant coupling type microstrip line interconnect using a bonding ribbon and dielectric pad. Both FDTD simulation and measurement have confirmed the design concept, and an insertion loss of better than $0.5 \mathrm{~dB}$ over a $40 \%$ bandwidth has been obtained for a low frequency model. The structure should be readily useful at higher frequencies as indicated by the full-wave simulation results. Furthermore, it is also shown that the structure can be easily expanded to high efficiency RF as well as DC level interconnection.

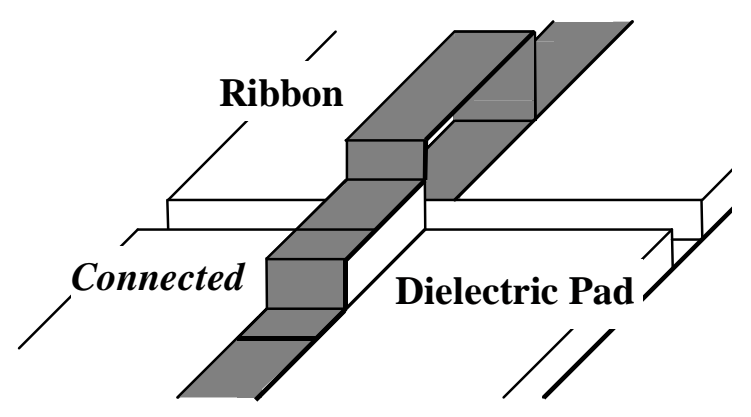

Fig. 6 Microstrip line interconnect with top conductor of dielectric pad and microstrip are connected.

\section{ACKNOWLEDGEMENT}

This work was supported by Rockwell MICRO.

\section{REFERENCES}

[1] W. Menzel, "Packaging and interconnects for millimeter wave circuits: a review," Ann. Telecommun., vol.51, no.3-4, pp.145154, 1997.

[2] G. Strauss and W. Menzel, "Millimeterwave monolithic integrated circuit interconnects using electromagnetic field coupling," IEEE Trans. Comp., Packag. and Manufact. Technol. - Part B, vol.19, no.2, pp.278-282, May 1996.

[3] H. Jin, R. Vahldieck, J. Huang and P. Russer, "Rigorous analysis of mixed transmission line interconnects using the frequency domain TLM method," IEEE Trans. Microwave Theory Tech., vol.41, no.12, pp.2248-2255, Dec. 1993.

[4] R. K. Hoffmann, "Microstrip line components", in Handbook of Microwave Technology; vol.1 (T. K. Ishii Ed.), San Diego, Academic Press Inc., 1995, Chap. 4.

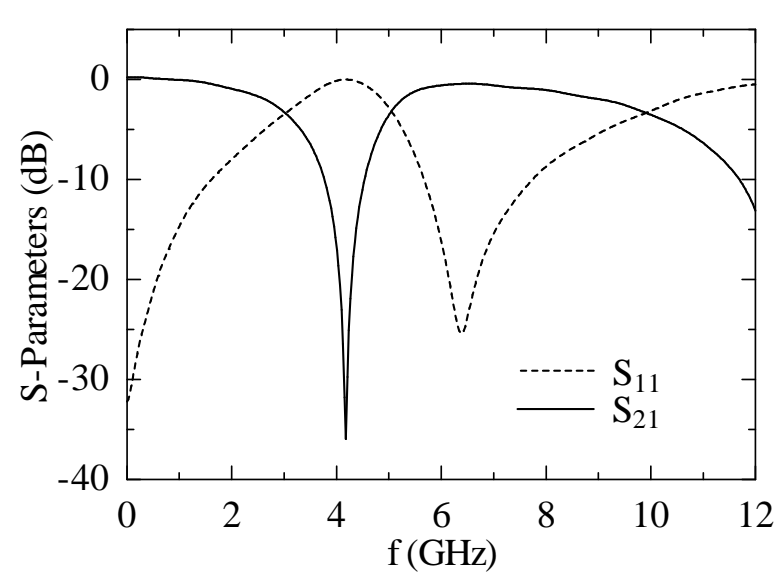

Fig. 7 S-parameters of the modified microstrip line interconnect. The parameters are the same as in Fig. 3. 